\title{
Spatially coordinated heterochromatinization of distal short tandem repeats in fragile $X$ syndrome
}

Linda Zhou ${ }^{1,3}$, Chunmin $\mathrm{Ge}^{1}$, Thomas Malachowski ${ }^{1}$, Ji Hun Kim¹, Keerthivasan Raanin

10 Chandradoss ${ }^{1}$, Chuanbin $\mathrm{Su}^{1}$, Hao $\mathrm{Wu}^{5}$, Alejandro Rojas ${ }^{5}$, Owen Wallace ${ }^{5}$, Katelyn R. Titus ${ }^{1}$, Wanfeng Gong ${ }^{1}$, Jennifer E. Phillips-Cremins ${ }^{1,2,3,4, *}$

\section{Affiliations:}

1 Department of Bioengineering, University of Pennsylvania, Philadelphia, PA

2 Epigenetics Institute, Perelman School of Medicine, University of Pennsylvania

3 Department of Genetics, Perelman School of Medicine, University of Pennsylvania

4 New York Stem Cell Foundation - Robertson Investigator

5 Fulcrum Therapeutics Incorporated, Cambridge, MA

*Corresponding author. Email: jcremins@seas.upenn.edu 


\begin{abstract}
$\underline{\text { Abstract }}$
Short tandem repeat (STR) instability is causally linked to pathologic transcriptional silencing in a subset of repeat expansion disorders. In fragile X syndrome (FXS), instability of a single CGG STR tract is thought to repress FMRI via local DNA methylation. Here, we report the acquisition of more than ten Megabase-sized H3K9me3 domains in FXS, including a 5-8 Megabase block around FMR1. Distal H3K9me3 domains encompass synaptic genes with STR instability, and spatially co-localize in trans concurrently with FMRI CGG expansion and the dissolution of TADs. CRISPR engineering of mutation-length FMRI CGG to normal-length preserves heterochromatin, whereas cut-out to pre-mutation-length attenuates a subset of $\mathrm{H} 3 \mathrm{~K} 9 \mathrm{me} 3$ domains. Overexpression of a pre-mutation-length CGG de-represses both FMRI and distal heterochromatinized genes, indicating that long-range $\mathrm{H} 3 \mathrm{~K} 9 \mathrm{me} 3$-mediated silencing is exquisitely sensitive to STR length. Together, our data uncover a genome-wide surveillance mechanism by which STR tracts spatially communicate over vast distances to heterochromatinize the pathologically unstable genome in FXS.
\end{abstract}

\title{
One-Sentence Summary
}

Heterochromatinization of distal synaptic genes with repeat instability in fragile $\mathrm{X}$ is reversible by overexpression of a pre-mutation length CGG tract. 


\section{$\underline{\text { Main Text }}$}

Fragile X syndrome (FXS) is the most common form of inherited intellectual disability, affecting 1 in 4,000 males and 1 in 8,000 females. The disease is made manifest early in life and presents as a range of mild to severe defects in communication skills, cognitive ability, and physical appearance, as well as hypersensitivity to stimuli, seizures, and anxiety (1). FXS is caused by expansion of a CGG STR tract in the 5' untranslated (5'UTR) region of the FMR1 gene (2). CGG tract length correlates with disease severity and can be stratified into <40 (normal-length), 41-55 (intermediate), 55-200 (pre-mutation), and 200+ (mutation-length) (3-7). Individuals with a premutation length CGG tract in FMRI can exhibit neurodevelopmental problems in their early years, and acquire late stage neurodegeneration due to Fragile $\mathrm{X}$-associated tremor/ataxia syndrome (FXTAS) (8). Moreover, in a process known as anticipation, mutation-length STRs grow longer as they are inherited across generations, leading to earlier onset and increased severity of FXS symptoms (9). These data highlight the critical role of precise STR tract lengths in a wide range of pathologic features during the onset and progression of human disease. in a number of repeat expansion disorders (10). In FXTAS, CGG expansion from normal-length to pre-mutation causes a 2-8-fold increase in FMRl expression, leading to pathologic nuclear inclusion bodies (8). By contrast, expansion from pre-mutation to mutation-length causes transcriptional inhibition of FMRI and consequent severe reduction in levels of the Fragile $\mathrm{X}$ Mental Retardation Protein (FMRP) it encodes $(11,12)$. Evidence to date suggests that transcriptional silencing occurs solely due to local DNA methylation and heterochromatinization of the FMRI CGG tract and its adjacent promoter $(13,14)$. Some genome-wide reports support this model by suggesting that pathologic changes to epigenetic modifications are restricted locally to FMR1 in FXS (15). However, DNA demethylation by 5-aza-2'-deoxycytidine treatment or 
direct targeting of dCas9-Tet1 only partially reinstates FMR1 transcription, and patient samples with longer CGG tracts are more refractory to FMRl de-repression (16-18). Moreover, recovery of FMRP levels through the use of human FMRl cDNA (19), artificial chromosomes (20), or viral vectors (21-23) cannot fully reverse FXS clinical presentations of synaptic plasticity, anxiety, seizure susceptibility, and macro-orchidism. Together, these data suggest that a subset of longterm pathologic features of FXS are made manifest independent from FMRP's downstream effects.

We recently reported severe local misfolding of the $3 \mathrm{D}$ genome around the $F M R 1$ gene in B cells and post-mortem brain tissue from FXS patients with a 450+ CGG STR expansion (24), suggesting that silencing might occur via long-range mechanisms beyond local DNA methylation. Here, we investigate the extent to which 3D chromatin architecture and linear epigenetic marks are altered genome-wide as a function of a gradient of CGG STR tract lengths. We analyzed a series of human induced pluripotent stem cell lines differentiated to neural progenitor cells (iPSCNPCs) in which the CGG STR tract is thought to expand from normal-length (5-30 CGG), premutation (130-190 CGG), short mutation-length (200-300 CGG), and long mutation-length (450+ CGG Replicate 1; 450+ CGG Replicate 2) (Fig. 1a). To obtain precise estimates of CGG STR length, we conducted a customized assay coupling Nanopore long-read sequencing with guide RNA-directed Cas9 cutting around the transcription start site and 5'UTR of the FMR1 gene (Fig. 1b-e, Fig. S1, Supplementary Methods, Table S1). Consistent with previous reports, wild type and pre-mutation lines had on average 34 and 160 total CGG STRs, respectively, with minimal interrupting sequences (Fig. 1b-e). FMRI mRNA increased upon expansion to the pre-mutation length as previously established (Fig. 1f). Unexpectedly, we observed that both short- and long mutation-length FXS lines showed a similar total of 425-460 CGG triplets (Fig. 1b). However, the short mutation length line contained a high number of AGG interrupters, leading to shorter and less continuous CGG tracts compared to long mutation-length (green, Fig. 1c-e). To facilitate 

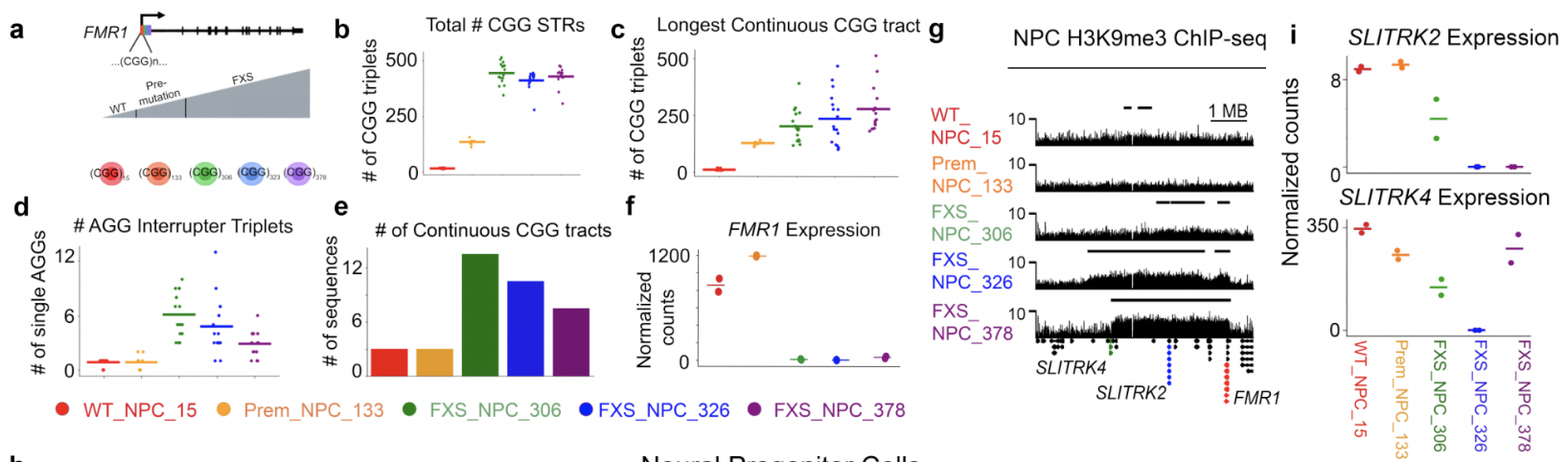

h

Neural Progenitor Cells
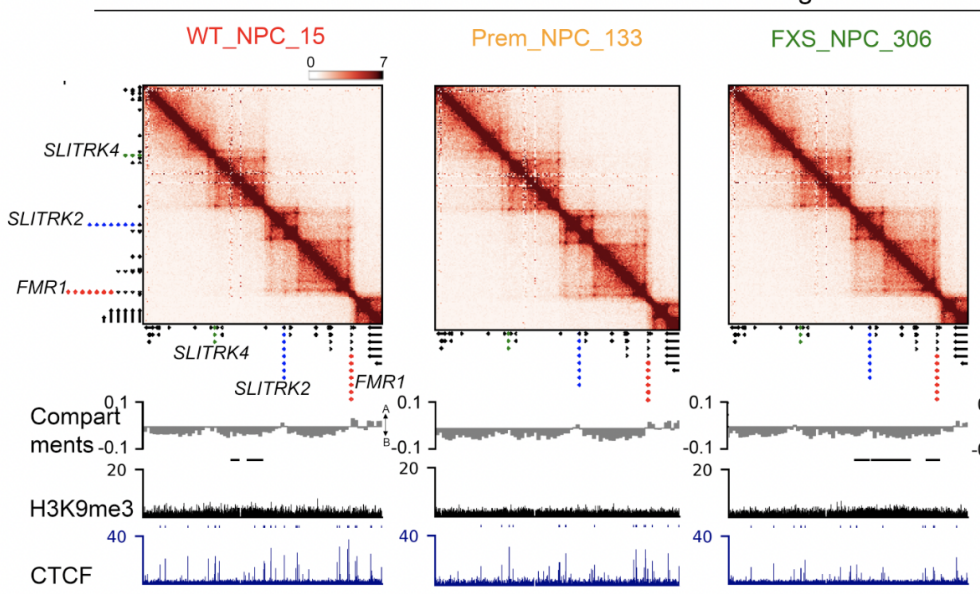

FXS_NPC_326

FXS_NPC 378
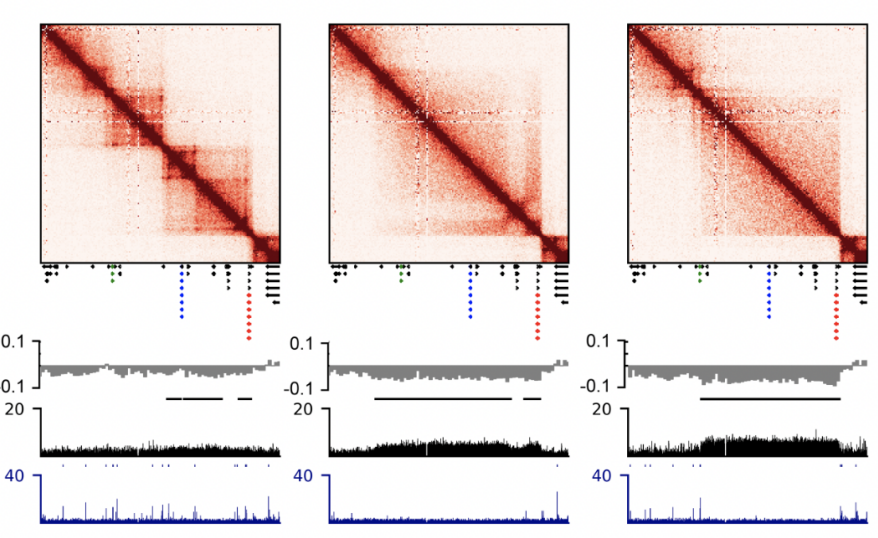

Figure 1. A >5 Megabase-sized domain of H3K9me3 heterochromatin spreads across the FMR1 locus in a CGG STR length-dependent manner in fragile $X$ syndrome.

(a) Schematic of iPSC lines used to model FXTAS and FXS, including normal-length, premutation length, short mutation-length, and long mutation-length. Colors are associated with each CGG length to identify STR sequence-dependent disease progression across all figures. (b-e) Nanopore long-read analysis of (b) total number of CGGs present, (c) longest continuous CGG tract, (d) number of AGG interrupters within CGG STR, and (e) total number of continuous CGG tracks within the STR in the 5'UTR of FMR1. (f) FMR1 mRNA levels as evaluated by RNA-seq. Horizontal lines represent the central tendency (mean) between $\mathrm{n}=2$ biological replicates. (g) $\mathrm{H} 3 \mathrm{~K} 9 \mathrm{me} 3 \mathrm{Chip}$-seq across all five lines is shown for an $8 \mathrm{Mb}$ region around FMR 1 . Gene track is plotted below ChIP-seq tracks. FMR1, SLITRK2, and SLITRK4 are highlighted in red, blue, and green respectively. (h) $\mathrm{Hi}-\mathrm{C}$ data across all five lines is shown as a heatmap of counts representing interaction frequency for an $8 \mathrm{Mb}$ region around FMR1. Compartment score, H3K9me3 Chip-seq, and CTCF Chip-seq in iPS-derived NPCs is displayed below the heatmaps for all five conditions. (i) SLITRK2 and SLITRK $4 \mathrm{mRNA}$ levels as evaluated by RNA-seq. Horizontal lines represent the central tendency (mean) between $\mathrm{n}=2$ biological replicates. 
clarity, we refer to the three FXS lines by the sum of their top two longest continuous CGG tracts - 306, 326, and 378 CGG triplets.

It is well established that AGG interrupters correlate with attenuated STR instability and decreased severity of disease (25), therefore we hypothesized that the FXS_306 short mutationlength line with a high frequency of interrupters would have less severe pathological defects to chromatin modifications than long mutation-length lines FXS_326 and FXS_378. We observed that FMRl gene expression decreased significantly to the same extent in all three FXS lines (Fig. 1f). Concomitant with decreased $F M R 1$, we observed increased DNA methylation around the transcription start site and the 5'UTR-localized CGG STR in all three FXS lines, suggesting that local levels of DNA methylation correlate strongly with the mRNA levels of FMRI (Fig. S2, Table S2). In stark contrast to local DNA methylation, we also observed CGG length-dependent acquisition of the repressive histone mark H3K9me3 (Fig. 1g). The gained H3K9me3 signal was not only local to FMR1 but spread upstream over $~ 3 \mathrm{Mb}$ in FXS_306 and increased in strength and spread further upstream to $>5 \mathrm{Mb}$ as the CGG tracts grew to 326 and $378 \mathrm{CGG}$ triplets (Fig. 1g-h, Table S3). Thus, the spread and intensity of a large H3K9me3 repressive heterochromatin domain correlates with the length of the continuous CGG tract, whereas local DNA methylation of the FMR1 promoter silences expression after the CGG passes short mutation-length.

We next studied the folding patterns of the 3D genome around the large acquired H3K9me3 domains (Table S4). In parallel with gained H3K9me3 (Fig. 1h, Fig. S3a-b), we observed strengthening of B compartment signal (Fig. 1h, Fig. S3a, S3c), loss of CTCF occupancy (Fig. 1h, Fig. S3d), and severe breakdown of TAD integrity (Fig. S3a, 3e) across the broader 5 Mbsized H3K9me3 domain. We also observed destruction of the local subTAD boundary at FMRI (Fig. S3f-h) as previously reported in B cells and post-mortem brain tissue (24). Our results 
demonstrate that heterochromatin silencing spreads more than $5 \mathrm{Mb}$ upstream of $F M R 1$ and correlates with severe large-scale misfolding of the 3D genome in FXS.

We noticed that the FXS H3K9me3 domain spanned two additional genes, SLITRK2 and SLITRK4, encoding known neuronal cell adhesion proteins linked to synaptic plasticity (Fig. 1gh). Expression of both SLITRK2 and SLITRK4 decreased in FXS in a manner that correlates with the spread of the H3K9me3 domain due to FMR1 CGG expansion (Fig. 1i). Using our Hi-C maps, we observed that FMR1 loops directly to SLITRK2 and SLITRK4 in wild type iPSC-NPCs containing a normal-length CGG STR tract (Fig. S3i-j). The long-range gene-gene cis interactions are abolished and SLITRK2 and SLITRK4 mRNA levels are decreased as H3K9me3 spreads over the locus in FXS (Fig. S3i-n, Fig. S4). We observed that SLITRK2/SLITRK4 are downregulated but not fully off in the FXS_NPC_306 line, suggesting that FMR1 silencing is governed by local DNA methylation whereas distal gene silencing is governed by general heterochromatin acquisition and 3D genome disruption (Fig. S3i-n). We also note that SLITRK4 is not silenced in one of the long mutation-length samples because the H3K9me3 domain does not extend up to the promoter of the gene, further emphasizing the likely functional role for $\mathrm{H} 3 \mathrm{~K} 9 \mathrm{me} 3$ in distal gene silencing in FXS (Fig. S5). Together, these data suggest that the acquisition of a large 5 Mb-sized H3K9me3 domain radiates outward from FMRl to encompass and silence additional synaptic genes as the mutation-length CGG STR further expands. FXS is characterized by clinical presentation of cognitive decline and defects in synaptic plasticity (26), so the direct spatial connection between FMRI and synaptic gene silencing is of critical importance toward understanding the onset of pathological features in the brain.

We wondered if our observations of large-scale 3D genome misfolding and heterochromatin silencing around the FMRI locus were specific to the NPC state. In pluripotent iPSCs, we observed the same pattern of large-scale H3K9me3 deposition gained with CGG STR 
expansion as in NPCs (Fig. S6). By contrast, in B cells our Hi-C analysis revealed that large scale genome folding disruptions did not occur upon mutation-length expansion (Fig. S7a-b). Importantly, the large $\mathrm{H} 3 \mathrm{~K} 9 \mathrm{me} 3$ domain is pre-existing in wild type B cells with the normal-length CGG tract, but stops at the TAD boundary before FMRI (Figs. S7-8). In mutation-length FXS B cells, the pre-existing H3K9me3 domain spreads over FMR1, and local CTCF occupancy and TAD boundary integrity are disrupted as we have previously reported (Fig. S7c-e) (24). Thus, 3D genome folding, CTCF occupancy, and H3K9me3-based heterochromatin silencing defects are cell type-specific in FXS and most severe in cell types such as NPCs where no pre-existing $\mathrm{H} 3 \mathrm{~K} 9 \mathrm{me} 3$ domain is present at the larger FMR1 locus. We next sought to understand if heterochromatin might be acquired on autosomes in FXS. We identified eleven additional genomic locations in which large ( $>1 \mathrm{Mb}) \mathrm{H} 3 \mathrm{~K} 9 \mathrm{me} 3$ domains were acquired with low signal in FXS_306 short mutation-length and subsequently strengthened and spread in FXS_326 and FXS_378 (Fig. 2a, Fig. S8, Tables S5-6). The same domains were present in iPSC (Fig. S9). One such domain encompasses the SHISA6 gene - a known fragile site on chromosome 17 (Fig. 2b). As seen at the broader FMRl locus, acquisition of H3K9me3 upon mutation-length CGG expansion occurs in parallel with TAD ablation and loss of CTCF occupancy (Fig. 2b-c). SHISA6 mRNA levels are decreased proportionately to the intensity of the H3K9me3 domain (Fig. 2d). Indeed, for all 11 distal FXS domains, we observed loss of CTCF occupancy (Fig. 2e, Fig. S10, Table S7), TAD boundary disruption (Fig. 2f, Fig. S10), and a marked reduction in gene expression (Fig. 2g, Fig. S11). Gene ontology analysis confirmed that genes in our FXS-specific H3K9me3 domains in iPSC-NPCs are involved in synaptic plasticity and neural cell adhesion, and such synaptic genes are not enriched in the H3K9me3 domains that are invariant across all CGG lengths (Fig. 2i-j, Fig. S12a, Table S8). We note that although we see both gain and loss of gene expression genome-wide in FXS (Fig. S13), it is only the 


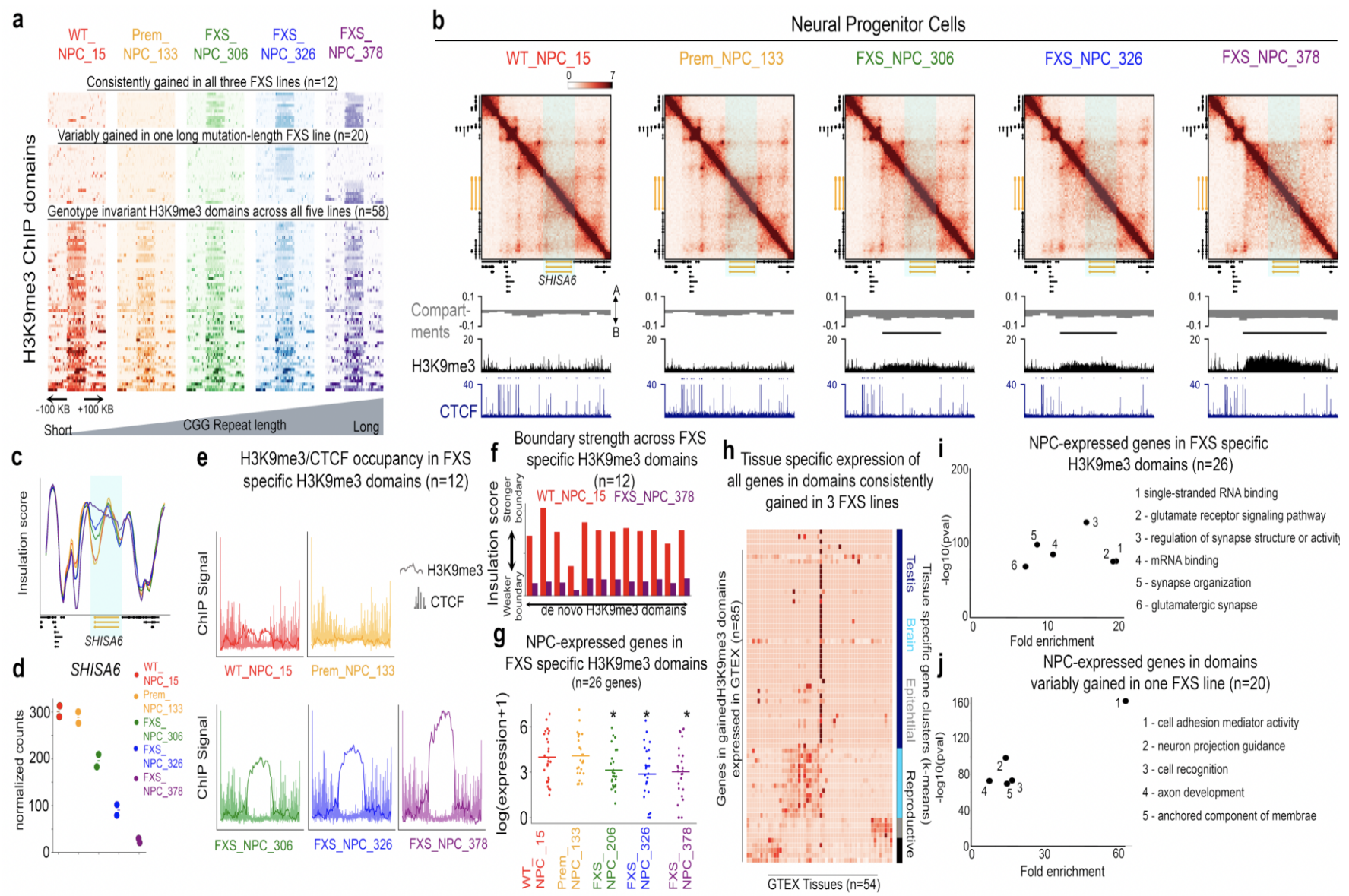

Figure 2: Acquisition of distal heterochromatin domains and silencing of key distal synaptic plasticity genes in fragile $\mathrm{X}$ syndrome.

(a) Three classes of H3K9me3 ChIP-seq domains identified genome-wide across five iPSC-derived NPC lines. H3K9me3 domains are defined as either (i) invariant across all genotypes, (ii) gained in FXS but not consistently in all disease lines, or (iii) consistently gained in all three FXS lines. Categorization was based on presence or absence of domains identified by RSEG. (b) Hi-C data across all five lines is shown as a heatmap of counts representing interaction frequency for a $1.6 \mathrm{Mb}$ region around one of the somatic H3K9me3 domains encompassing SHISA6. Compartment score, H3K9me3 ChIP-seq, and CTCF ChIP-seq in iPSC-NPCs is displayed below the heatmaps for all five conditions. Lines representing RSEG H3K9me3 domain calls are show above H3K9me3 ChIP-seq track. SHISA6 gene is highlighted in orange. (c) Insulation score across $\mathrm{Hi}-\mathrm{C}$ matrices in the same $1.6 \mathrm{Mb}$ region as (b) for all five cell lines. Red, orange, green, blue, purple correspond to normal-length, pre-mutation, short mutation-length, long mutation-length sample 1, and long mutation-length sample 2, respectively. SHISA6 gene is highlighted in orange. (d) SHISA6 mRNA levels as evaluated by RNA-seq. Horizontal lines represent the central tendency (mean) between $\mathrm{n}=2$ biological replicates. (e) Pooled H3K9me3 and CTCF ChIP-seq data across all $n=12$ consistently gained H3K9me3 domains in FXS. (f) Insulation score for the strongest domain boundary in each of the $\mathrm{H} 3 \mathrm{~K} 9 \mathrm{me} 3$ domains consistently gained in all three FXS lines for WT_NPC_15 (red) and FXS_NPC_378 (purple) cell lines. There are 12 sets of one red and one purple bar plot, each set corresponding to one H3K9me3 domain. (g) mRNA levels as evaluated by RNA-seq for $\mathrm{n}=26$ protein coding genes in consistently gained domains in FXS. Genes are only shown if they were expressed in at least one cell line. Each point represents expression of one gene averaged across $n=2$ biological replicates. * indicates $\mathrm{p}<0.05$ when compared to WT_NPC_15. Pvalues 
were calculated using a one-tailed Mann Whitney U test. (h) Expression of all genes in consistently gained domains in FXS across tissues in the GTEX dataset. Genes were only shown if expression was not 0 across all tissues, resulting in $n=67$ genes. Genes were clustered using K-means clusters into 4 groups, and clusters were labelled based on the tissue types dominating each cluster. (i-j) Gene ontology (GO) analysis using WebGESTALT for (i) $n=26$ protein coding genes expressed in iPS-derived NPCs and localized to $\mathrm{H} 3 \mathrm{~K} 9 \mathrm{me} 3$ domains consistently gained in $\mathrm{FXS}$ and $(\mathbf{j}) \mathrm{n}=20$ protein coding genes expressed in iPS-derived NPCs and localized to H3K9me3 domains gained in FXS but not consistently in all FXS lines as defined in panel (a).

downregulated genes in our NPC H3K9me3 domains that exhibit synaptic gene ontology (Fig. S12a-c). In addition to $\mathrm{N}=12$ heterochromatin domains present across all FXS cell lines, we also identified $20 \mathrm{H} 3 \mathrm{~K} 9 \mathrm{me} 3$ domains specific to just one cell line (Fig. 2a, 2j), indicating that heterogeneity in clinical presentation in FXS patients may be due to different distributions of heterochromatinization. Together, our data reveal that large H3K9me3 domains also arise distal from the FMRI locus and encompass genes critically linked to the synaptic plasticity defects characteristic of FXS (27).

Macro-orchidism and soft skin are unexplained clinical presentations in FXS (28), and expansion of the FMRl CGG STR also causes severe ovary defects in Fragile X-associated primary ovarian insufficiency (FXPOI) (29). To understand the transcriptional profile of our H3K9me3-localized genes in tissues outside the brain, we examined their expression across 54 tissues from the GTEX consortium. We observed that genes localized to FXS heterochromatin domains largely exhibit tissue-specific expression profiles, including testis, female reproductive organs, epithelium, and (consistent with our NPC results) brain (Fig. 2h, Fig. S14). Given that our NPC FXS-specific H3K9me3 domains are also present in iPSCs, these results suggest that many of such domains will also be present in skin and reproductive tissues and thus relevant to the silencing of genes linked to non-brain pathology. Our results bring to light a compelling hypothesis in which distal heterochromatinization and silencing of epithelial and testis genes on autosomes is 
a mechanism contributing to pathological features outside the brain in a broad range of clinical presentations due to FMR1 CGG instability.

Given that the primary site of STR expansion is in FMRI on the $\mathrm{X}$ chromosome, it remains quite unusual that large distal genomic loci would be heterochromatinized in FXS. To understand how FMR1 communicates with distal loci, we examined Hi-C trans matrices (Supplementary Methods). We unexpectedly observed unusually strong trans (i.e. inter-chromosomal) interactions connecting the FMR1 locus specifically to distal H3K9me3 domains (Fig. 3a-b, Fig. S15). Importantly, all distal silenced H3K9me3 domains form a multi-way subnuclear hub with FMRI in FXS (Fig. 3c). We observed that the formation of trans interactions occurs concomitant with the density of H3K9me3 acquired during disease progression (Figs. S15-20). Trans interactions are not present in normal-length or pre-mutation length. They initiate upon short mutation-length (FXS_306) expansion, and form full interaction strength as the H3K9me3 domains spread in long mutation-length (FXS_326, FXS_378) (Figs.S15-20). Together, these data show that the genomewide gained FXS heterochromatin domains engage directly via spatial proximity with the unstable FMR1 locus upon mutation-length expansion of the CGG STR tract.

Heterochromatinization is known to protect the repetitive genome against instability (30). We hypothesized that genes in FXS H3K9me3 domains would require spatially coordinated heterochromatinization because they fall in genomic locations that are highly susceptible to instability. Consistent with this idea, we first noticed that the majority of our FXS domains overlapped established human fragile sites (Fig. 3d, Fig. S21). We also noticed that, like FMR1, nearly all of the FXS-specific distal H3K9me3 domains are located at the ends of chromosomes adjacent to sub-telomeric regions (Fig. 3e). Using high-coverage whole genome PCR-free sequencing and the GangSTR computational method (31), we quantified STR tract length genome- 

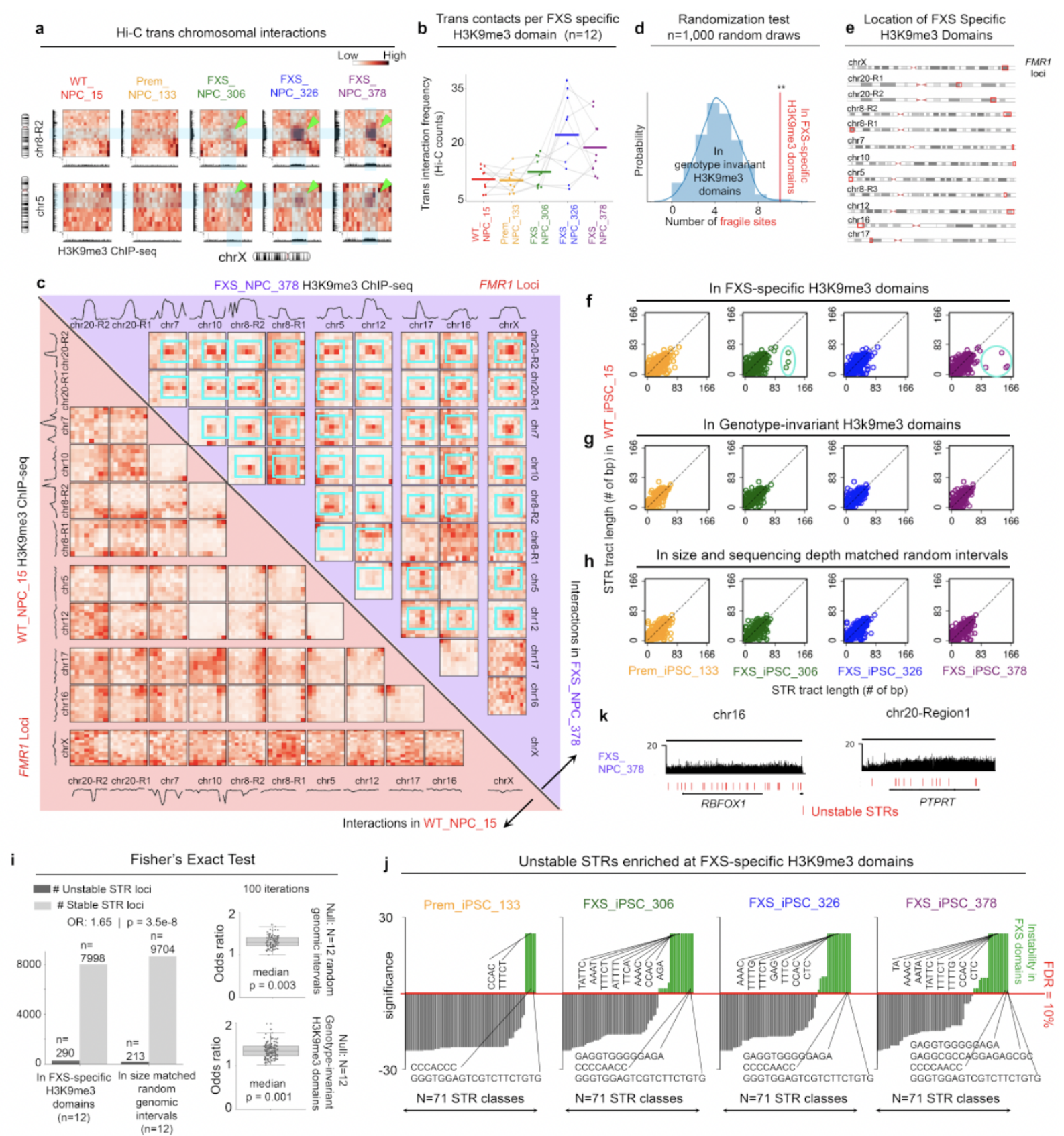

Figure 3: FXS heterochromatin domains form a spatial subnuclear hub of trans interactions between distal genes exhibiting short tandem repeat instability.

(a) Hi-C inter-chromosomal interaction heatmaps binned at $1 \mathrm{Mb}$ resolution between FMR1 H3K9me3 domains and H3K9me3 domains on chromosome 8 and chromosome 5. The window for each region includes the $\mathrm{H} 3 \mathrm{~K} 9 \mathrm{me} 3$ domain gained in $\mathrm{FXS}$ and $5 \mathrm{Mb}$ of flanking genome. H3K9me3 Chip-seq is shown on the $\mathrm{x}$ - axis for chromosome $\mathrm{X}$ and for the $\mathrm{y}$-axis for the distal region. Blue bars and green arrows highlight trans interactions. (b) Interchromosomal contacts between all FXS H3K9me3 domains and the FMRl domain on chromosome X. Each dot represents one gained H3K9me3 domain. Lines connecting the dots show the progress of that domain across 5 cell lines with increasing CGG STR length. Bar represents mean trans contacts across all domains. (c) Pairwise Hi-C interactions among all of 
the distal H3K9me3 domains gained in FXS for long mutation-length (FXS_NPC_378, upper triangle) and normal-length (WT_NPC_15, lower triangle). Domains annotated by chromosome. The window for each region includes the H3K9me3 domain gained and $3 \mathrm{Mb}$ of flanking genome. H3K9me3 Chip-seq signal for all domains for both FXS_NPC_378 and WT_NPC_15 are plotted above Hi-C heatmaps. Blue boxes highlight FXS gained trans interactions. (d) The number of fragile sites within the H3K9me3 domains in FXS compared to a null distribution consisting of 1000 draws of $n=12$ size-matched, randomly-sampled intervals located within genotype-independent $\mathrm{H} 3 \mathrm{~K} 9 \mathrm{me} 3$ domains that remain constant throughout normal-length, short mutation, premutation, and disease lines. as called by RSEG. Fragile sites were obtained from the HumCFS database. (e) The location of the gained H3K9me3 domain at FMRI and n=11 distal gained H3K9me3 domains is highlighted in a red box on a chromosome ideogram obtained from the UCSC genome browser. (f) The length of STR types (in bp) in FXS-specific H3K9me3 domains which differ by at least 3 units in at least 1/3 disease lines compared to WT are plotted. WT_NPC_15 length is plotted on the y-axis and remaining cell lines are plotted on the x-axis. (g) Same as f, for STRs in genotype-invariant H3K9me3 domains. (h) Same as f, but for random genomic intervals matched to FXS-specific H3K9me3 domains in size and sequencing depth. (i) Fisher's Exact test comparing the number of stable vs unstable STR loci in FXS specific H3K9me3 domains vs an equal number coverage matched genome invariant H3K9me3 domains. The results of a 1000 Fisher's exact tests, run on 1000 different sets of randomly drawn size matched genomic intervals or genome invariant H3K9me3 domains are also shown. (j) Results of a statistical test (see Supplemental Methods) demonstrating which of the $\mathrm{N}=71$ classes of STRs which are unstable in FXS are enriched for instability in FXS-specific H3K9me3 domains vs genome invariant H3K9me3 domains. An FDR of 10\% was used as a threshold for significance. The individual N=71 loci are plotted on the $\mathrm{x}$-axis from least to most significant. The $\mathrm{y}$-axis, significance, is $10 * \log (\mathrm{BH}$-corrected p-value + pseudocount $)$, centered so that FDR of less than $10 \%$ is positive and the remaining are negative. (k) H3K9me3 and unstable STRs are shown for the RBFOX1 gene and the PTPRT gene.

wide across our healthy and FXS iPSC lines (Table S9). We observed that a small subset of STR types are indeed expanding or contracting specifically in our FXS disease iPSC lines compared to normal-length wild type iPSCs (Fig. 3f, Fig. S22, Supplementary Methods). Importantly, pathologically unstable STR tracts are highly enriched in our FXS-specific H3K9me3 domains compared to random size-matched genomic intervals or genotype-invariant H3K9me3 domains

(Fig. 3f-h, Fig. 3i, Fig. S23a, Supplementary Methods). Together, this work demonstrates that regions of the genome silenced in FXS are similar to FMRl loci in that they are at the ends of 
chromosomes and are enriched for pathologically unstable STRs. We posit that instability may predispose distal STR loci as targets of the same mechanisms driving H3K9me3 deposition at the larger FMR1 locus.

To further understand why the unstable FMRl locus would spatially contact and coordinate heterochromatinization with our specific distal locations and not with other locations in the genome, we explored the genetic features of unstable STRs in our FXS H3K9me3 domains. We formulated a statistical test (Supplementary Methods) to identify specific STR tracts which are expanding at higher-frequency in our FXS-specific H3K9me3 domains compared to random sizematched genomic intervals or genotype-invariant H3K9me3 domains (Fig. 3j, Fig. S23b). A list of top pathologically unstable STRs enriched in FXS-specific H3K9me3 domains are provided (Tables S11-S13). Distal CGG STR tracts did not noticeably expand, but this could be due to inaccurate GangSTR estimates on high CG-content tracts (Fig. S23c). Importantly, genes localized in FXS-specific H3K9me3 domains are significantly longer and exhibit a significantly higher density of pathologically unstable STR tracts per gene compared to the null expectation of all genes in random size-matched genomic intervals or even genotype-invariant H3K9me3 domains present across all five lines (Fig. 3k, Fig. S23d-e). Together, our data inspired a working model in which STR tracts across the genome communicate with each other spatially via trans interactions as a surveillance mechanism that enables the heterochromatinization and silencing of STR tracks at risk of instability.

To understand the functional role of the FMRl CGG STR in heterochromatin deposition in cis and in trans, we examined the extent of $\mathrm{H} 3 \mathrm{~K} 9 \mathrm{me} 3$ reversibility after shortening the CGG to pre-mutation or normal-length with CRISPR (Fig. 4a). In our first iPSC cohort, we cut back the FMR1 CGG tract in the long mutation-length FXS_iPSC_378 line to the normal-length range of 4 CGG triplets as confirmed by Nanopore long-reads (Fig. 4a-b, Figs. S24-25). We observed that 
the large H3K9me3 domain spanning SLITRK4, SLITRK2, and FMR1 did not notably change after CGG STR cut-back to normal-length (Fig. 4c). CTCF binding was not re-gained and genome folding domains remained destroyed just as in the FXS_iPSC_378 parent line (Fig. S26). Consistent with previous reports, the FMRI gene was partially de-repressed in the normal-length cut-out, however SLITRK2 remained silenced (Fig. 4d). We also noticed that all distal heterochromatinized loci maintained a high level of H3K9me3 signal upon normal-length CGG cut-out (Fig. S27). Our data indicate that engineering the CGG STR back to normal-length range does not markedly reprogram $\mathrm{H} 3 \mathrm{~K} 9 \mathrm{me} 3$ domains in cis or in trans, suggesting that pathologically silenced synaptic, epithelial, testis, and female reproductive tissue genes will not be de-repressed with an FMRI CGG normal-length cut-out strategy in FXS.

In our second iPSC cohort, we cut back the FMRl CGG tract in the long mutation-length FXS_iPSC_326 line to a pre-mutation length of 180 CGG triplets, as confirmed by Nanopore sequencing (Fig. 4a-b, Figs. S24-25). We unexpectedly observed that the H3K9me3 domain encompassing SLITRK4, SLITRK2, and FMR1 is fully reversible upon cut-out to premutationlength (Fig. 4c). Corroborating the loss of H3K9me3, CTCF occupancy was re-gained and TAD boundaries were re-instated at the broader FMR1 locus (Fig. 4e). Both SLITRK2 and FMR1 mRNA levels were nearly fully restored upon engineering to pre-mutation length (Fig. 4d). Our results suggest that the reversal of the $\mathrm{H} 3 \mathrm{k} 9 \mathrm{me} 3$ heterochromatin domain around $F M R 1$ might require a step back through the stage of disease acquisition involving the pre-mutation length CGG STR.

We next queried the extent to which the distal H3K9me3 domains in FXS could be reversed upon local FMRI CGG STR engineering. By contrast to the cut-out to normal-length range where no distal H3K9me3 signal was altered, we observed that a subset of distal H3K9me3 domains were fully reprogrammed upon only engineering of the FMRI CGG STR precisely to 180 CGG pre- 
a
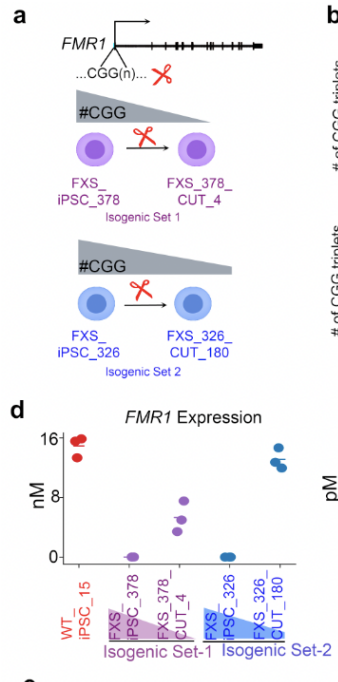

e
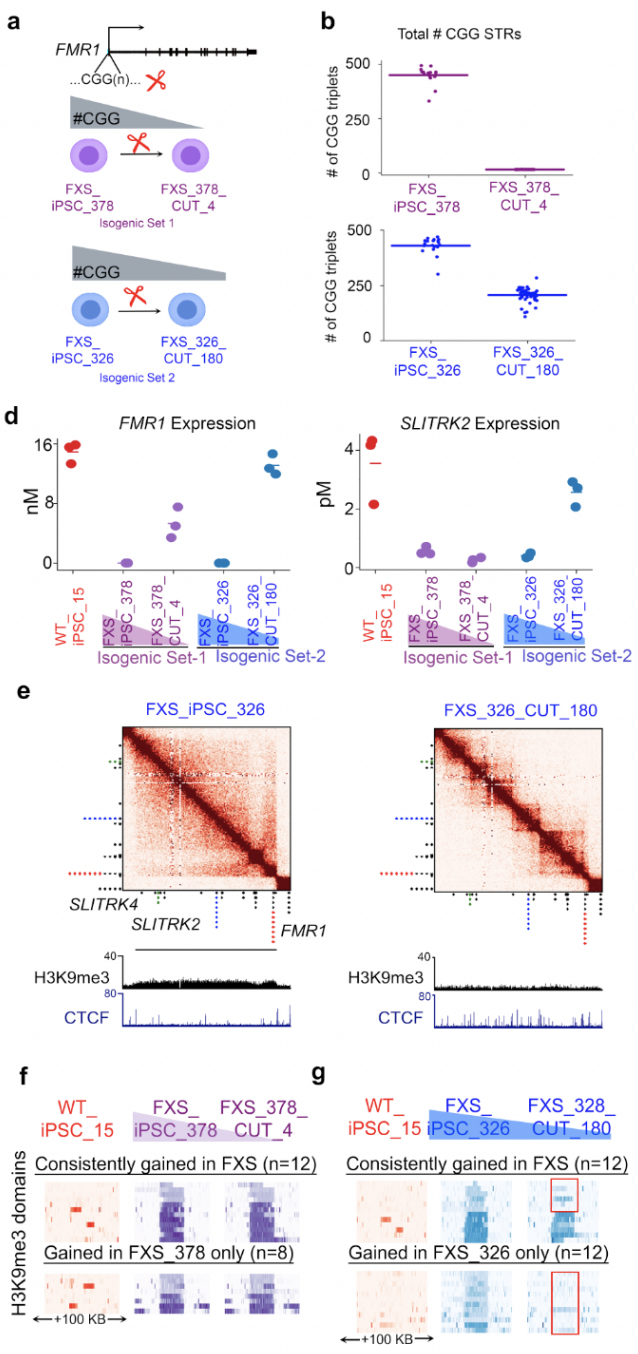

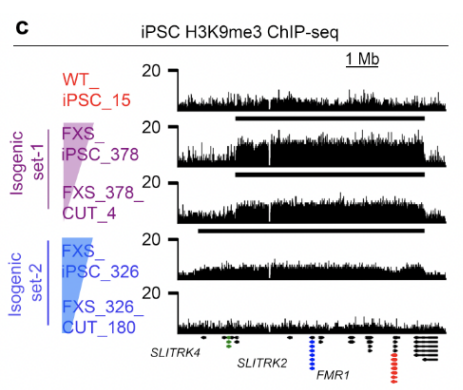

h

i

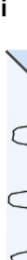

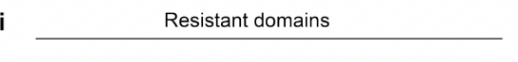

Reprogrammed domains

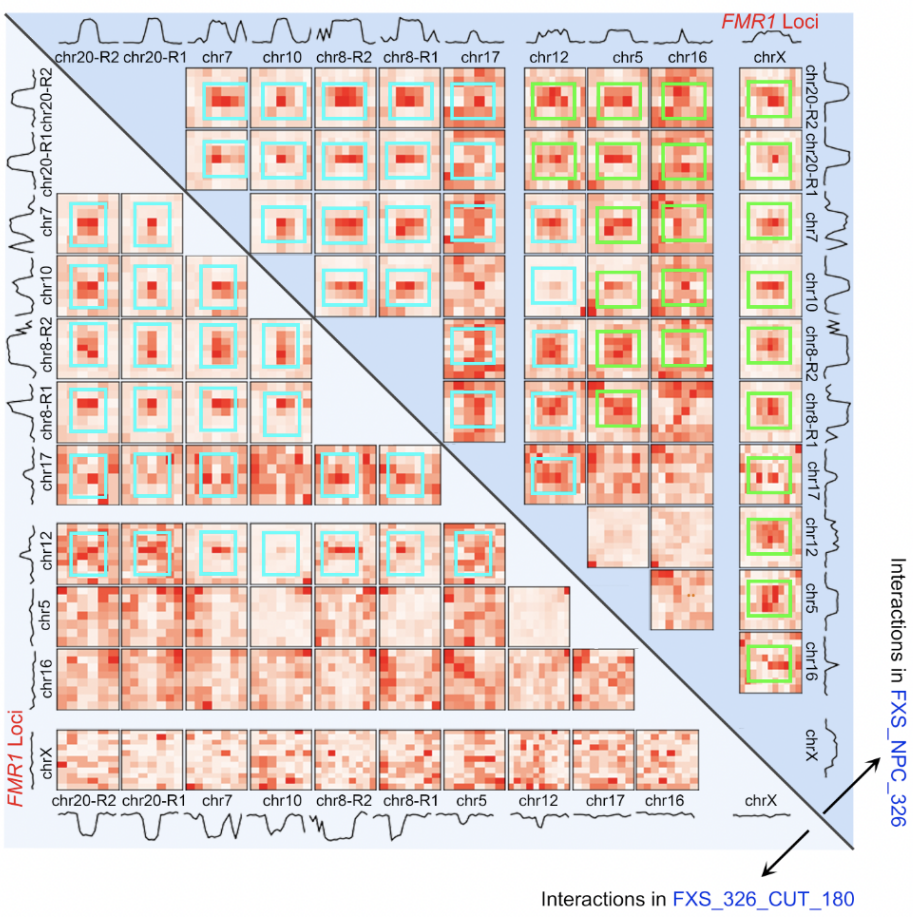

Retained in FXS_326_CUT_180 Lost in FXS 326 CUT 180

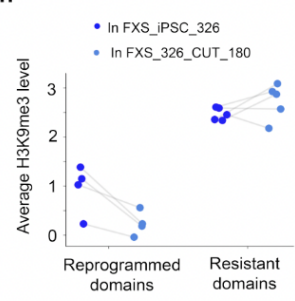

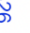

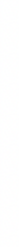


signal is shown for each of $n=12$ heterochromatin domains consistently gained across all three FXS lines as well as heterochromatin domains present only in the FXS parent line for iPSC in (f) isogenic set 1 and (g) isogenic set 2. Each line of the heat map represents one region. Red boxes annotate reprogrammed domains that lose H3K9me3 signal upon FMRl CGG STR shortening. (h) Average H3K9me3 signal in isogenic set 2 (FXS_iPSC_326 and FXS_326_CUT_180) for each FXS H3K9me3 domain, stratified up by whether the domain was reprogrammed or resistant upon shortening of the mutation-length CGG to pre-mutation length. (i) Pairwise Hi-C interactions among all of the distal H3K9me3 domains gained in FXS for long mutation-length (FXS_iPSC_326, upper triangle) and pre-mutation length (FXS_180, lower triangle). Domains annotated by chromosome. The window for each region includes the H3K9me3 domain gained and $3 \mathrm{Mb}$ of flanking genome. H3K9me3 ChIP-seq signal for all domains for both lines are plotted above Hi-C heatmaps. Blue boxes highlight FXS trans interactions that are resistant to reprogramming. Green boxes highlight FXS trans interactions that are reprogrammed upon CGG shortening to pre-mutation length.

mutation length (Fig. 4f-g, Fig. S27). Distal domains with the lowest H3K9me3 density were the most susceptible to reprogramming after engineering the FMRl CGG STR (Fig. 4h). Although the majority of distal high-density H3K9me3 loci remained tethered in a trans interaction hub, the FMRI locus and several distal domains lost their heterochromatinization and spatially disconnected upon engineering of the mutation-length CGG at FMRl to pre-mutation (Fig. 4i, Fig. S28). Together, these results highlight the remarkable ability of the FMRI CGG STR to communicate spatially in trans with distal $\mathrm{H} 3 \mathrm{~K} 9 \mathrm{me} 3$ domains, functionally contributing, at least in part, to the acquisition of their pathologic heterochromatinization. Importantly, reverse engineering of the FMRl CGG to pre-mutation length can fully reverse the H3K9me3 domain locally at FMR1 and attenuate a subset of distal H3K9me3 domains. The persistence of heterochromatin silencing at many reprogramming resistant $\mathrm{H} 3 \mathrm{~K} 9 \mathrm{me} 3$ domains in $\mathrm{FXS}$ highlights the importance of additional clinical interventions beyond FMRI CGG STR engineering, and suggests that many distal $\mathrm{H} 3 \mathrm{~K} 9 \mathrm{me} 3$ domains in FXS may form through a mechanism that is independent of the FMRl CGG. 
Finally, we sought to understand if overexpression of a pre-mutation CGG STR sequence alone, independent from its placement in the FMRI gene, was sufficient to attenuate local or distal FXS H3K9me3 domains. We queried gene expression and H3K9me3 after overexpressing a transgene expressing 99 CGG triplets (pre-mutation) in long mutation-length FXS iPSCs for 48 hours (Fig. 5a). We observed a striking de-repression of FMR1, SLITRK2, DPPA6, and SHISA6, with a much higher effect size than that observed due to CRISPR CGG engineering to pre-mutation length within the endogenous FMR1 locus (Fig. 5b-e, Fig. S29). Using CUT\&RUN for H3K9me3, which is amenable to assaying signal in low cell numbers, we observed complete ablation of nearly all distal H3K9me3 heterochromatin domains in FXS upon overexpression of the pre-mutation CGG STR (Fig. 5f-h). Altogether, these data reveal that both local and distal heterochromatin domain acquisition in FXS can be fully reversed by ectopic expression of a pre-mutation length CGG STR, suggesting that the spatial subnuclear hub of fragile repetitive regions in FXS is driven by a CGG-mediated DNA or RNA mechanism that transcends FMRl.

Altogether, our data support a model in which we find pervasive long-range transcriptional silencing in FXS via the acquisition of a physically connected subnuclear hub of more than ten Megabase-sized domains of the repressive histone modification H3K9me3. Such domains acquire low levels of $\mathrm{H} 3 \mathrm{~K} 9 \mathrm{me} 3$ signal in the transition from pre-mutation to short mutation-length and increase in severity and spread of $\mathrm{H} 3 \mathrm{~K} 9 \mathrm{me} 3$ density as the FMRl CGG STR expands to long mutation-length (Fig. 5i). Consistent with previous reports, we see that local DNA methylation of the FMRI gene correlates with its degree of silencing. By contrast, a large cohort of genes distal from FMRI are repressed in FXS in a manner commensurate with the severity of $\mathrm{H} 3 \mathrm{~K} 9 \mathrm{me} 3$ deposition. It has long been thought that global gene expression disruption in FXS is due to the downstream effects of FMRP loss, however here we see that the CGG STR expansion in FMRI activates a genome-wide surveillance system to deposit large H3K9me3 domains to directly 
silence STR-rich genes localized at the ends of distal chromosomes. The FXS pathologic heterochromatin domains encompass and silence genes critical for synaptic plasticity, testis development, female reproductive system functioning, and epithelial tissue structure, which are directly related to the clinical presentations in FXS. Our results suggest that pharmacological and RNA-based interventions to reverse distal H3K9me3 silencing may provide tangible therapeutic benefits to FXS patients if genome stability can be maintained.

It is difficult to envision how a CGG STR expansion event in FMRI could coordinate heterochromatinization on 10 other chromosomes. Here, we see evidence of a physically linked subnuclear hub of inter-chromosomal interactions among known human fragile sites and long genes with high density of unstable short tandem repeat tracts in FXS. We hypothesize that critical areas of the genome communicate to coordinate silencing when instability events are detected. CRISPR engineering of the long mutation-length CGG tract to pre-mutation length provides evidence that at least a subset of distal domains is heterochromatinized and spatially connected in a manner that depends on the length of the FMRI CGG STR. We hypothesize that the DNA sequence or RNA encoded by the pre-mutation CGG STR tract will functionally contribute to the reversal of FXS heterochromatinization, as we demonstrate that overexpression of a generic premutation-length CGG STR transgene results in complete attenuation of all distal H3K9me3 domains and full de-repression of distal genes. It is noteworthy that CRISPR shortening of the mutation length CGG STR to normal-length only slightly de-represses FMRI and has no noticeable effect on distal heterochromatin domains. Other studies showing stronger FMRI derepression upon local CGG cut-out to normal-length may have started with a shorter mutationlength tract with lower density of $\mathrm{H} 3 \mathrm{~K} 9 \mathrm{me} 3$ signal more amenable to reprogramming $(32,33)$. Our results suggest that genetically engineering approaches relying only on cut-out of the FMR 1 


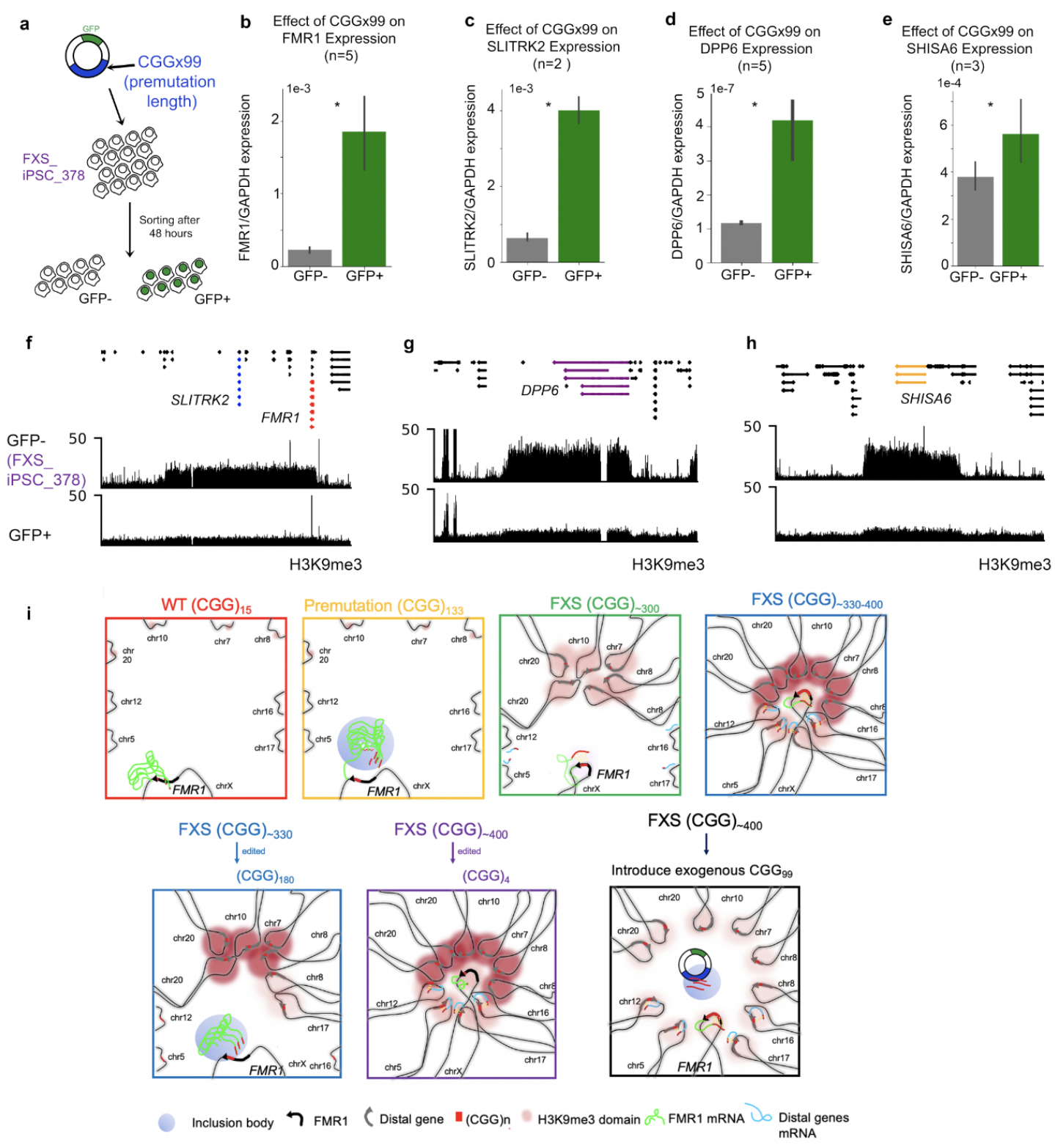

Figure 5: Overexpression of a pre-mutation length CGG STR tract de-represses pathologically silenced expression and attenuates FXS H3K9me3 domains.

Schematic showing experimental workflow. ( b-e) mRNA levels as assessed by qRT-PCR for (b) FMR1, (c) SLITRK2, (d) DPP6, and (e) SHISA6 in long mutation-length FXS iPSCs which either did receive (GFP+) or did not receive (GFP-) the CGGx99 plasmid. Error bars represent the standard error of the mean for the indicated number of technical replicates. (f-h) H3K9me3 CUT\&RUN in either the GFP- or GFP+ cells at the gained H3K9me3 domains at (f) FMR1, (g) DPP6, and (h) SHISA6. (i) Schematic model. We find that numerous heterochromatin domains interact via long-range trans interactions to form an inter-chromosomal subnuclear hub with the FMRI locus in fragile X syndrome. When CGG STRs are normal-length, FMRI and other chromosomes do not cluster and do not interact in trans. As the CGG tract expands to premutation length, FMRI mRNA levels increase. When the CGG STR tract expands to short mutation-length, FMRI expression drastically decreases. Distal fragile sites acquire large H3K9me3 domains that cluster together spatially in trans. When CGG repeats expand to long 
mutation-length (450), FMR1 mRNA levels are fully repressed, and the distal heterochromatin domains gain $\mathrm{H} 3 \mathrm{~K} 9 \mathrm{me} 3$ signal intensity. Upon cutout from long mutation-length to premutation, a subset of distal domains loses $\mathrm{H} 3 \mathrm{~K} 9 \mathrm{me} 3$ signal and the long-range trans interactions with FMR1 are abolished. By contrast, cutout of long mutation-length to normal-length CGG triplets does not reverse heterochromatin domains, trans interactions remain connected, and genes remain repressed. Finally, the role for the pre-mutation length CGG tract is made evident upon introduction of an exogenous 99 CGG triplet STR transgene to FXS iPSCs. The presence of transcribed CGG plasmid leads to reduction of heterochromatin across all gained H3K9me3 domains and reactivates FMRI and distal gene expression, suggesting that long-range 3D Epigenome miswiring in FXS is driven by the DNA or RNA CGG STR sequence.

CGG may not reverse the silencing of key genes contributing to persistent pathology in FXS patients. Full attenuation of pathologic features across multiple tissues may require combination therapies coupling pharmacological intervention targeting epigenetic writers and erasers, as well as STR engineering. Altogether, our work uncovers a pervasive genome-wide surveillance mechanism by which fragile sites and STR tracts in the human genome spatially communicate over vast distances to heterochromatinize and silence the unstable genome. 


\section{$\underline{\text { Acknowledgments }}$}

We thank members of the Cremins lab for helpful discussions, in particular Kenneth Pham and Zoltan Simandi for critical feedback and Michael Guo for assistance with GangSTR. Jennifer E. Phillips-Cremins is a New York Stem Cell Foundation - Robertson Investigator and an Alfred P.

Sloan Foundation Fellow. Linda Zhou is a Blavatnik Family Fellow.

\section{References}

1. M. R. Santoro, S. M. Bray, S. T. Warren, Molecular mechanisms of fragile X syndrome: a twenty-year perspective. Annu Rev Pathol 7, 219-245 (2012).

2. A. R. La Spada, H. L. Paulson, K. H. Fischbeck, Trinucleotide repeat expansion in neurological disease. Ann Neurol 36, 814-822 (1994).

3. S. M. Mirkin, Expandable DNA repeats and human disease. Nature 447, 932-940 (2007).

4. D. L. Nelson, H. T. Orr, S. T. Warren, The unstable repeats--three evolving faces of neurological disease. Neuron 77, 825-843 (2013).

5. A. R. La Spada, J. P. Taylor, Repeat expansion disease: progress and puzzles in disease pathogenesis. Nat Rev Genet 11, 247-258 (2010).

6. C. T. McMurray, Mechanisms of trinucleotide repeat instability during human development. Nat Rev Genet 11, 786-799 (2010).

7. C. E. Pearson, K. Nichol Edamura, J. D. Cleary, Repeat instability: mechanisms of dynamic mutations. Nat Rev Genet 6, 729-742 (2005).

8. R. J. Hagerman, P. Hagerman, Fragile X-associated tremor/ataxia syndrome - features, mechanisms and management. Nat Rev Neurol 12, 403-412 (2016).

9. R. I. Richards et al., Evidence of founder chromosomes in fragile X syndrome. Nat Genet 1, 257-260 (1992).

10. H. T. Orr, H. Y. Zoghbi, Trinucleotide repeat disorders. Annu Rev Neurosci 30, 575-621 (2007).

11. H. Y.Zoghbi, M. F. Bear, Synaptic dysfunction in neurodevelopmental disorders associated with autism and intellectual disabilities. Cold Spring Harb Perspect Biol 4, (2012).

12. A. Contractor, V. A. Klyachko, C. Portera-Cailliau, Altered Neuronal and Circuit Excitability in Fragile X Syndrome. Neuron 87, 699-715 (2015).

13. J. S. Sutcliffe et al., DNA methylation represses FMR-1 transcription in fragile X syndrome. Hum Mol Genet 1, 397-400 (1992).

14. Y. Zhou, D. Kumari, N. Sciascia, K. Usdin, CGG-repeat dynamics and FMR1 gene silencing in fragile X syndrome stem cells and stem cell-derived neurons. Mol Autism 7, 42 (2016).

15. R. S. Alisch et al., Genome-wide analysis validates aberrant methylation in fragile X syndrome is specific to the FMR1 locus. BMC Med Genet 14, 18 (2013).

16. B. Coffee, F. Zhang, S. T. Warren, D. Reines, Acetylated histones are associated with FMR1 in normal but not fragile X-syndrome cells. Nat Genet 22, 98-101 (1999). 
17. B. Coffee, F. Zhang, S. Ceman, S. T. Warren, D. Reines, Histone modifications depict an aberrantly heterochromatinized FMR1 gene in fragile x syndrome. Am J Hum Genet 71, 923-932 (2002).

18. X. S. Liu et al., Rescue of Fragile X Syndrome Neurons by DNA Methylation Editing of the FMR1 Gene. Cell 172, 979-992 e976 (2018).

19. S. A. Musumeci et al., Audiogenic seizure susceptibility is reduced in fragile X knockout mice after introduction of FMR1 transgenes. Exp Neurol 203, 233-240 (2007).

20. A. M. Peier et al., (Over)correction of FMR1 deficiency with YAC transgenics: behavioral and physical features. Hum Mol Genet 9, 1145-1159 (2000).

21. S. Gholizadeh, J. Arsenault, I. C. Xuan, L. K. Pacey, D. R. Hampson, Reduced phenotypic severity following adeno-associated virus-mediated Fmr1 gene delivery in fragile X mice. Neuropsychopharmacology 39, 3100-3111 (2014).

22. Z. Zeier et al., Fragile X mental retardation protein replacement restores hippocampal synaptic function in a mouse model of fragile X syndrome. Gene Ther 16, 1122-1129 (2009).

23. J. Arsenault et al., FMRP Expression Levels in Mouse Central Nervous System Neurons Determine Behavioral Phenotype. Hum Gene Ther 27, 982-996 (2016).

24. J. H. Sun et al., Disease-Associated Short Tandem Repeats Co-localize with Chromatin Domain Boundaries. Cell 175, 224-238 e215 (2018).

25. E. E. Eichler et al., Length of uninterrupted CGG repeats determines instability in the FMR1 gene. Nat Genet 8, 88-94 (1994).

26. M. Telias, Molecular Mechanisms of Synaptic Dysregulation in Fragile X Syndrome and Autism Spectrum Disorders. Front Mol Neurosci 12, 51 (2019).

27. B. E. Pfeiffer, K. M. Huber, The state of synapses in fragile X syndrome. Neuroscientist 15, 549-567 (2009).

28. J. F. Atkin, K. Flaitz, S. Patil, W. Smith, A new X-linked mental retardation syndrome. Am J Med Genet 21, 697-705 (1985).

29. H. Tan, H. Li, P. Jin, RNA-mediated pathogenesis in fragile X-associated disorders. Neurosci Lett 466, 103-108 (2009).

30. A. Janssen, S. U. Colmenares, G. H. Karpen, Heterochromatin: Guardian of the Genome. Annu Rev Cell Dev Biol 34, 265-288 (2018).

31. N. Mousavi, S. Shleizer-Burko, R. Yanicky, M. Gymrek, Profiling the genome-wide landscape of tandem repeat expansions. Nucleic Acids Res 47, e90 (2019).

32. N. Xie et al., Reactivation of FMR1 by CRISPR/Cas9-Mediated Deletion of the Expanded CGG-Repeat of the Fragile X Chromosome. PLoS One 11, e0165499 (2016).

33. C. Y. Park et al., Reversion of FMR1 Methylation and Silencing by Editing the Triplet Repeats in Fragile X iPSC-Derived Neurons. Cell Rep 13, 234-241 (2015). 